\title{
Mujer anciana con diarrea crónica
}

\author{
Elderly woman with chronic diarrhea
}

\author{
Laura González Vázquez¹, Alberto Fernández Villaverde², Fernando González Carrili3, Javier de la Fuente Aguado. \\ ${ }^{1}$ Servicio de Medicina Interna, ${ }^{2}$ Servicio de Aparato Digestivo, ${ }^{3}$ Servicio de Anatomía Patológica. \\ Hospital POVISA. Vigo
}

\begin{abstract}
Resumen
La enfermedad celiaca típicamente se diagnostica en niños y adultos jóvenes con historia de diarrea o malabsorción. Sin embargo, cada vez es más frecuente detectar esta patología en mayores de 55 años. Por este motivo, nos ha parecido interesante describir un nuevo caso en una paciente anciana y realizar una breve actualización.
\end{abstract}

Palabras clave. Enfermedad celiaca. Anciano. Complicaciones.

\section{Introducción}

La enfermedad celiaca (EC) es un proceso autoinmune producido por una intolerancia permanente al gluten y que aparece en personas genéticamente predispuestas (HLA-DQ2 y HLA-DQ8)'. Se genera una inflamación crónica en la mucosa del intestino delgado, la cual altera la absorción de algunos nutrientes. Hasta hace pocos años, la EC era considerada una enfermedad poco frecuente, de diagnóstico habitualmente en la infancia, donde suele presentarse con la tríada clásica de diarrea, distensión abdominal y retraso de crecimiento 4 . En adultos, el espectro de manifestaciones clínicas es muy variable, y abarca desde formas completamente asintomáticas hasta cuadros clínicos muy diversos, complejos y de amplio diagnóstico diferencial.

\section{Caso clínico}

Mujer de 75 años que acude por cambio en su ritmo intestinal consistente en diarrea acuosa de unas 5-7 deposiciones al día, sin productos patológicos, desde hacía unos 40 días, sin respetar el sueño nocturno. Se acompañaba de urgencia e incontinencia. No asociaba fiebre ni dolor abdominal. Previamente tendencia al estreñimiento con una deposición de características normales cada 2 ó 3 días. Había perdido peso pero no apetito. No refería antecedentes epidemiológicos ni familiares de relevancia. Como antecedentes personales presentaba HTA, dislipemia, y vejiga hiperactiva. Había sido intervenida de prótesis de rodilla derecha, hernia discal L4-L5, e histerectomía simple. Recibía tratamiento con loperamida, calcio oral, oxibutinina y valsartan.

En la exploración física la TA era de 130/80 mmHg, la frecuencia cardiaca 70 latidos por minuto y la temperatura de $36,8^{\circ} \mathrm{C}$. No tenía signos de deshidratación y presentaba una coloración normal de piel y mucosas. La auscultación cardiopulmonar no presentaba ninguna alteración y en la exploración abdominal tan solo se apreciaba una cicatriz de laparotomía media infraumbilical, sin otra alteración relevante. Se realizó una analítica de sangre que mostró una hemoglobina de $12,8 \mathrm{gr} / \mathrm{dl}$ con un hematocrito del $40 \%$ y un volumen corpuscular medio normal. El número de leucocitos así como la fórmula leucocitaria estaban en rangos normales. La glucosa, urea, creatinina, ácido úrico, colesterol total y LDL, y las proteínas totales fueron normales. La GOT era de 54

\section{Abstract}

Celiac disease is typically diagnosed in children and young adults with a history of diarrhea or malabsorption. However, this disease is increasingly detected in patients older than 55 years. For this reason, it seemed interesting to describe a new case in an elderly patient and to make a brief update.

Key words. Coeliac disease. Elderly. Complications.

UI/L (4-37 UI/L), la GPT de $40 \mathrm{UI} / \mathrm{L}$ (4-38 UI/L)), la GGT de $49 \mathrm{UI} / \mathrm{L}$ (4-50 UI/L) con una bilirrubina normal, un hierro de $48 \mu \mathrm{g} / \mathrm{dl}(40-150 \mu \mathrm{g} / \mathrm{dl})$ y una ferritina de $41 \mathrm{ng} / \mathrm{ml}(10-100 \mathrm{ng} / \mathrm{ml})$. Se realizó una TSH basal que fue de $2.07 \mu \mathrm{Ul} / \mathrm{ml}$; la sangre oculta en heces determinada en 3 ocasiones fue negativa. Los anticuerpos antiendomisio IgA resultaron positivos con un título de 1/320 y los antitransglutaminasa IgA también fueron positivos (294,1 AU/ml).

Los hallazgos macroscópicos de la gastroscopia fueron normales excepto por una leve gastritis crónica. Se tomaron biopsias de segunda y tercera porción duodenal donde se apreció inflamación crónica con linfocitosis intraepitelial, hiperplasia de criptas y atrofia vellositaria parcial, compatibles con enfermedad celiaca en estadio IIIA de la clasificación modificada de Marsh (Figura1). Una colonoscopia mostró la presencia de grandes divertículos y descartó colitis microscópica. Se eliminó el gluten de la dieta con buen cumplimiento y evolución clínica favorable, desapareciendo la diarrea y aumentando el peso, con importante mejoría del estado general y posterior normalización de la serología.

\section{Discusión}

La enfermedad celiaca (EC) está producida por una intolerancia permanente al gluten presente en alimentos como la cebada, el centeno 0 el trigo ${ }^{1}$. Su prevalencia en la población española está alrededor del 0,45-0,85\% en la población pediátrica y $0,26 \%$ en la adulta ${ }^{2}$. Se considera una enfermedad de la infancia y de adultos jóvenes, aunque en distintos estudios se ha puesto de manifiesto que hasta el $20 \%$ de los pacientes tiene más de 60 años ${ }^{3} y$ en mayores de 70 años presenta una mayor prevalencia en varones. En adultos, y especialmente en ancianos, suele existir un retraso diagnóstico debido a la presencia de manifestaciones atípicas y a que está alterada la historia natural de la enfermedad. La secuencia de eventos (aparición de anticuerpos, desarrollo de la enteropatía intestinal, comienzo de los síntomas y, finalmente, desarrollo de las complicaciones) no necesariamente tiene que ocurrir en cada paciente ${ }^{4}$. Tampoco está claro que una determinación de anticuerpos negativa sea suficiente para excluir la EC, ya que la seroconversión puede ocurrir años después del inicio de los 
síntomas, como ha demostrado Vilppula et al en mayores de 55 años 5 . Por tanto, la susceptibilidad genética y la ingesta de gluten en la dieta son necesarios pero no suficientes para desarrollar la enfermedad celiaca ${ }^{4}$.

Las alteraciones más frecuentes en el caso de los adultos son la diarrea (55\%), la anemia ferropénica (48\%) y la elevación de transaminasas $(38 \%)^{6}$. La forma de presentación en ancianos no difiere de los adultos jóvenes aunque parece que están asintomáticos en un mayor porcentaje ${ }^{3}$.

Con respecto a la diarrea, debemos considerar que los pacientes con EC tienen multiplicado por 70 el riesgo de padecer colitis microscópica si los comparamos con la población general ${ }^{7}$, por lo que se recomienda que sean sometidos a cribado serológico para descartar $\mathrm{EC}^{3}$. Además, el $27 \%$ de los casos de colitis linfocítica se asocian con EC y parece que presenta también asociación con la colitis colágena, siendo en algunos estudios de hasta un $40 \%{ }^{8}$. Por este motivo, en caso de mala respuesta a la dieta sin gluten, se deben descartar estas entidades.

En cuanto a las alteraciones hematológicas, la ferropenia puede ser la única manifestación y algunos autores propugnan su uso como marcador del cumplimiento de la dieta libre de gluten 0 para detectar casos de ingestión inadvertida 9 . También se ha observado frecuentemente trombocitopenia y en pacientes ancianos, se ha descrito algún caso de trombocitosis sin otra causa aparente que la $\mathrm{EC}^{10}$. Además, la gastritis atrófica es más frecuente en pacientes ancianos con EC que en jóvenes y el déficit asociado de factor intrínseco puede producir malabsorción de vitamina B12. Así, el déficit de B12 se ha visto en el 21\% de los mayores de 70 años con EC y en el $37 \%$ de los mayores de 80 años ${ }^{11}$. Por otro lado, se ha descrito déficit de vitamina $\mathrm{D}$ hasta en el $66 \%$ de los pacientes ${ }^{12}$ que se asocia a un mayor riesgo de fracturas, aunque debido a la gran heterogeneidad en los estudios, se recomienda investigar de una manera más amplia ${ }^{13}$

También se ha asociado la EC con un riesgo dos veces mayor de padecer enfermedades malignas, especialmente linfomas no Hodgkin, tanto de células B como T, con un pico de incidencia en la séptima década de la vida. Parece que este riego no está aumentado en EC latente ${ }^{14}$. Más dudosa es su asociación con tumores sólidos en distintas localizaciones (esófago, páncreas, intestino delgado, colon, vía biliar) ${ }^{1}$ ya que el riesgo no va más allá del primer año de seguimiento ${ }^{14}$.

La EC también se ha asociado con demencia en menores de sesenta años y, al parecer, en pacientes con deterioro cognitivo leve, éste puede ser revertido mediante una dieta estricta sin gluten ${ }^{3}$. Esta retirada del gluten en los pacientes diagnosticados en la edad adulta provoca una mejoría significativa en síntomas gastrointestinales que podrían incluso haber pasado desapercibidos para el paciente ${ }^{15}$. Así, un estudio publicado recientemente evalúa el efecto beneficioso que la dieta aporta en pacientes mayores de 50 años que habían sido diagnosticados mediante cribado serológico poblacional apreciando una mejoría subjetiva significativa en el GSRS (Gastrointestinal Symptom Rating Scale), y objetiva en los niveles de ferritina, de hemoglobina en mujeres, de vitamina B12, de vitamina $D$ y $E^{15}$. En esta serie también llama la atención que el $23 \%$ de los pacientes tenían una historia de fracturas de baja energía y el 29\% estaban diagnosticados de otras enfermedades autoinmunes, por lo que recomiendan realizar cribado en estos subgrupos ${ }^{15}$. En el caso de los pacientes

Figura 1. Biopsia duodenal: inflamación crónica con linfocitosis intraepitelial, hiperplasia de criptas y atrofia vellositaria parcial (compatibles con EC en estadio IIIA de la clasificación modificada de Marsh)

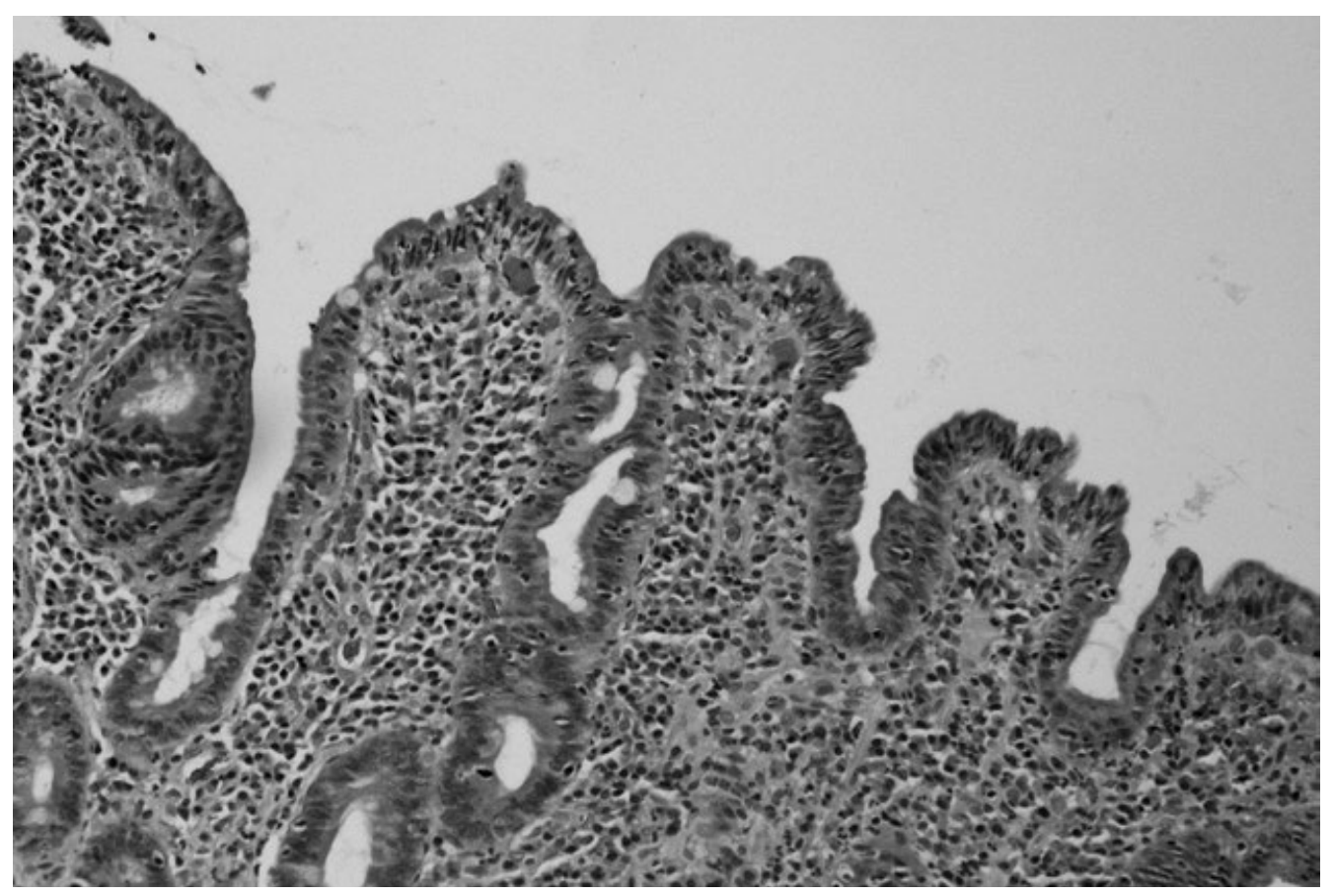


ancianos diagnosticados de EC y en los que la diarrea sea persistente, además de valorar el adecuado cumplimiento de la dieta, debemos descartar insuficiencia pancreática, la cual mejora al administrar suplementos enzimáticos ${ }^{16}$.

Se recomienda el seguimiento anual de estos pacientes para revisar su adherencia a la dieta, con monitorización serológica y de complicaciones que se pueden asociar, principalmente osteoporosis y enfermedad autoinmune tiroidea ${ }^{4}$. La positividad de anticuerpos IgA anti-transglutaminasa, o su recurrencia tras haberse negativizado, suelen indicar mal cumplimiento dietético.

Conviene tener en cuenta que en los pacientes con EC, tanto niños como adultos, la prevalencia de déficit de IgA puede ser hasta 15 veces mayor que en la población general ya que ambas presentan una fuerte asociación con la misma región HLA (DQ2) ${ }^{17}$. Por ello, se recomienda determinar la $\lg A$ y, si existe déficit, solicitar anticuerpos del tipo lgG antitransglutaminasa. Recientemente, se ha introducido como alternativa la determinación de anticuerpos IgG anti-péptidos deaminados de la gliadina, los cuales tienen mayor sensibilidad y especificidad que los IgA anti-transglutaminasa y harían innecesario el cribado de $\lg A^{4}$.

En resumen, en pacientes mayores de 55 años con diarrea crónica, elevación de transaminasas, defectos vitamínicos, osteopenia u osteoporosis establecida con fracturas de baja energía, se debe excluir la presencia de EC mediante la realización de anticuerpos IgA anti-transglutaminasa (con determinación simultánea de (gA), que son los que presentan mayor sensibilidad y especificidad. Si el resultado es positivo, está indicada la realización de gastroscopia con toma de biopsias duodenales.

\section{Bibliografía}

1. Green PH, Cellier C. Celiac disease. N Engl J Med 2007; 357:1731-43.

2. Riestra Menéndez S. Epidemiología de la enfermedad celíaca. En: Enfermedad celíaca. Introducción al conocimiento de la enfermedad celíaca. Ergon; 2011. p. 25-32.

3. Johnson MW, Ellis HJ, Asante MA and Ciclitira PJ. Celiac disease in the elderly. Nat Clin Pract Gastroenterol Hepatol. 2008 Dec; 5(12):697-706.

4. Fasano A, Catassi C. Celiac Disease. N Engl J Med 2012; 367:2419-26.

5. Vilppula A, Kaukinen K, Luostarinen L, Krekela I, Patrikainen H, Valve R, Mäki M, Collin P. Increasing prevalence and high incidence of celiac disease in elderly people: A population-based study. BMC Gastroenterology 2009, 9:49.

6. Fernández A, González L, de la Fuente J. Coeliac disease: clinical features in adult populations. Rev Esp Enferm Dig 2010; 102: 466-471.

7. Green PH, Yang J, Cheng J, Lee AR, Harper JW, Bhagat G. An association between microscopic colitis and celiac disease. Clin Gastroenterol Hepatol 2009; 7:1210-6.

8. Guillett HR and Freeman HJ. Prevalence of coeliac disease in collagenous and lymphocytic colitis. Can J Gastroenterol. 2000 Dec; 14(11):919-21.

9. Baccini F, Spiriti MA, Vannella L, Monarca B, Delle Fave G, Annibale B. Unawareness 0 gastrointestinal symptomatology in adult coeliac patients with unexplained iron-deficiency anaemia presentation. Aliment Pharmacol Ther. 2006 Apr 1; 23(7):915-921.

10. Carroccio A, Giannitrapani L, Di Prima L, lannitto E, Montalto G, Notarbartolo A. Extreme thrombocytosis as a sign of coeliac disese in the elderly: case report. Eur $\mathrm{J}$ Gastroent Hepatol 2002; 14(8): 897-900.

11. Kassarjian Z and Russell RM. Hypochlorhydria: a factor in nutrition. Ann Rev Nutr 1989 (9):271-285.

12. Meyer D, Stavropolous S, Diamond B, Shane E, Green PH. Osteoporosis in a North American adult population with celiac disease. Am J Gastroenterol 2001; 96(1):112119.

13. Olmos M, Antelo M, Vázquez H, Smecuol E, Maurino E, Bai JC. Systematic review and meta-analysis of observational studies on the prevalence of fractures in coeliac disease. Dig Live Dis 2008 Jan; 40(1): 46-53.

14. Elfström P, Granath F, Ekström Smedby K, Montgomery SM, Askling J, Ekbom A Ludvigsson JF. Risk of lymphoproliferative malignancy in relation to small intestinal histopathology among patients with celiac disease. J Natl Cancer Inst. 2011 Mar 2; 103(5):436-44.

15. Vilppula A, Kaukinen K, Luostarinen L, Krekela I, Patrikainen H, Valve R, Luostarinen $M$, Mäki M, Collint P. Clinical benefit of gluten-free diet in screen-detected older celiac disease patients. BMC Gastroenterology 2011 Dec 16; 11:136.

16. Leeds JS, Hopper AD, Hurlstone DP, Edwards SJ, McAlindon ME, Lobo AJ, Donnelly MT, Morley S, Sanders DS. Is exocrine pancreatic insufficiency in adult coeliac disease a cause of persisting symptoms? Aliment Pharmacol Ther. 2007; 25(3): 265-271.

17. Wang N, Shen N, Vyse TJ, Anand V, Gunnarson I, Sturfelt G, Rantapää-Dahlqvist S, Elvin K, Truedsson L, Andersson BA, Dahle C, Ortqvist E, Gregersen PK, Behrens TW, Hammarström L. Selective IgA deficiency in autoimmune diseases. Mol Med. 2011; 17(11-12):1383-96. 